\title{
Ausdifferenzierung von SwissDRG soll Hochdefizitfälle reduzieren
}

\author{
Petra Ingenpass ${ }^{a}$, Barbara Rohner \\ a Dr. med., Stv. Leiterin Abteilung Tarife und Gesundheitsökonomie Spitalärzte \\ ${ }^{b}$ Wissenschaftliche Mitarbeiterin Abteilung Tarife und Gesundheitsökonomie Spitalärzte
}

Die sachgerechte Abbildung hochdefizitärer Fälle steht bei der Weiterentwicklung von SwissDRG seit Jahren auf der Agenda. Die SwissDRG AG hat nun vier Lösungsvorschläge geprüft und einen Grundsatzentscheid gefällt: Lösungen zur aufwandsgerechteren Vergütung von Hochdefizitfällen sollen auch künftig innerhalb der Tarifstruktur gesucht werden - unter Einbezug von Fällen mit Überdeckung.

\section{Fälle mit deutlicher Unter- aber auch Überdeckung}

SwissDRG ist ein pauschalierendes Entgeltsystem, das medizinisch und ökonomisch vergleichbare Behandlungsfälle in einer Fallpauschale zusammenfasst und den durchschnittlichen Aufwand vergütet. Abweichungen zwischen Erlös und tatsächlichem Ressourcenverbrauch sind systemimmanent. Im Idealfall gleichen sich Unter- und Überdeckung von Behandlungsfällen innerhalb eines Spitals aus. In der Realität sind jedoch vor allem hochdefizitäre Fälle asymmetrisch auf die Spitäler verteilt und es ist aus Sicht der SwissDRG AG kaum möglich, dass auf Spitalebene alle Verlust- durch Gewinnfälle kompensiert werden können. Davon betroffen sind insbesondere Universitätsspitäler, Kinderspitäler oder Erbringer hochkomplexer medizinischer Leistungen. Hochdefizitäre Fälle entstehen aufgrund besonderer Konstellationen - die Behandlung ist so aufwendig und kostenintensiv, dass die verbrauchten Ressourcen nicht annähernd durch den Erlös aus der Fallpauschale abgedeckt werden.

\section{Vier Lösungsansätze standen zur Diskussion}

Die SwissDRG AG ist sich der Problematik hochteurer Behandlungsfälle bewusst und hat diese bereits in ihre Entwicklungsstrategie 2013+ aufgenommen. Die Arbeitsgruppe Hochdefizitfälle der SwissDRG AG hat deshalb vier Vorschläge zur aufwandsgerechteren Vergütung solcher Fälle vorgelegt.
Lösungsansatz 1: Die Tarifstruktur SwissDRG soll bei der jährlichen Weiterentwicklung so ausdifferenziert werden, dass hochdefizitäre Fälle nach Möglichkeit vermieden werden. Dazu müssten zum einen sämtliche Kalkulationsvorgaben überprüft werden wie z.B. die Differenzkostenmethodik, die Kalkulationsmethode für Outlier oder die Berechnung der Kostengewichte ausschliesslich auf Grundlage der Kosten von Inliern. Zum anderen ist zu prüfen, ob Konstellationen spezifischer Merkmale von Hochdefizitfällen mit Hilfe von Zusatzentgelten vergütet werden können. Hochdefizitäre Fälle sind häufig mit langer Aufenthaltsdauer, dem Kriterium "zuverlegt», Geburtsgebrechen oder einem Aufenthalt auf einer Intensivstation assoziiert. Solche Merkmale gilt es zu definieren.

Lösungsansatz 2: Dieser Vorschlag sieht die Lösung in der Differenzierung von Baserates für die Spitäler, die einen überdurchschnittlichen Anteil hochdefizitärer Fälle aufweisen - d.h. Universitätsspitäler und Kliniken mit komplexem und speziellem Leistungsportfolio. Festzulegen sind objektive Kriterien für die Differenzierung der Baserate. Dabei gilt es, nicht nur die Anzahl an Hochdefizitfällen und das Defizitvolumen, sondern auch die Hochgewinnfälle zur Vermeidung von Verzerrungen zu berücksichtigen. Sollten diese Massnahmen nicht ausreichen, um Wettbewerbsnachteilen von Leistungserbringern mit höherer Baserate entgegenzuwirken, wären weitere Schritte zu prüfen. Bei Lösungen ausserhalb der Tarifstruktur (Varianten 3 und 4) sind im gesamtschweizerisch gültigen und vom Bundesrat genehmigten Tarifstrukturvertrag vorgängig folgende Fragen zu klären: Was ist ein Hoch- 
defizitfall? Wie ist die Zusatzfinanzierung definiert? Welche Kriterien müssen erfüllt sein, damit ein Spital eine zusätzliche Finanzierung für seine Hochdefizitfälle erhält? Für eine Zusatzfinanzierung werden Vorgaben zur Datenqualität, zum maximalen Volumen der zusätzlichen Abgeltung oder auch zum Verhältnis von Gewinn- und Verlustfällen definiert. Eine vollständige Deckung des Defizits ist nicht vorgesehen, Spitäler müssen weiterhin einen Teil der Unterdeckung selbst tragen. Die Lösungsvarianten drei und vier hätten eine Wechselwirkung zwischen der Zusatzfinanzierung sowie der zu verhandelnden Baserate zur Folge. Zudem ist die KVG-Konformität dieser beiden Varianten zu prüfen.

Lösungsansatz 3: Für jeden Hochdefizitfall erfolgt eine Zusatzabgeltung durch die Kostenträger. Der maximale Deckungsgrad des Einzelfalles wird vorgängig im Tarifstrukturvertrag definiert.

Lösungsansatz 4: Dieser Vorschlag sieht Zahlungen aus einem Pool vor. Alle Spitäler mit Hochdefizitfällen können demnach ihre Ansprüche geltend machen. Die Speisung des Pools könnte beispielsweise über einen solidarischen Fallbeitrag erfolgen.

\section{FMH plädiert für eine Ausdifferenzierung von SwissDRG}

Die FMH unterstützt den Ansatz, Lösungen zur aufwandsgerechteren Vergütung hochdefizitärer Fälle KVG-konform innerhalb der Tarifstruktur SwissDRG zu suchen. Im Rahmen der jährlichen Weiterentwicklung von SwissDRG konnte bereits eine Verbesserung in der Abbildung solcher Fälle erzielt werden - nicht zuletzt durch die Schaffung von mehr Zusatzentgelten. Diesen Weg gilt es aus Sicht der FMH weiter zu verfolgen und Ausdifferenzierungen im Rahmen der strukturellen Möglichkeiten zu suchen. Wichtig dabei ist, entsprechende Entwicklungen des G-DRG-Systems auf Kompatibilität und Nutzen für SwissDRG zu prüfen. Beispielsweise wurde im G-DRG-System die Gesamtfallschwere zur besseren Abbildung weniger, hochteurer Fälle erweitert. ${ }^{1}$ Aber auch Zusatzentgelte für spezifische Merkmale hochdefizitärer Fälle sieht die FMH als praktikablen Ansatz an, der weiter verfolgt werden soll.

Bis die für Hochdefizitfälle notwendige Ausdifferenzierung der Tarifstruktur Wirkung zeigt, sind differenzierte Baserates unvermeidlich. Die FMH hatte sich bereits bei der Einführung von SwissDRG für differenzierte Baserates nach Spitalkategorie als Übergangslösung zum Ausgleich der noch ungenügenden Tarifstruktur ausgesprochen. Dauerhaft angewendete differenzierte Baserates lösen das Kernproblem aber nicht, sondern verzerren den Wettbewerb zulasten von
Spitälern mit einem Leistungsauftrag für hochkomplexe und spezialisierte Medizin. Zudem erschweren differenzierte Baserates nicht nur die Tarifverhandlungen, sie sind gemäss Entscheid des Bundesverwaltungsgerichtes auch nur während der Einführungsphase von SwissDRG KVG-konform. ${ }^{2}$

Die Mehrheit der medizinischen Fachgesellschaften und Dachverbände ist der Ansicht, dass der Lösungsansatz $3 \mathrm{zu}$ komplex ist und ausserdem noch viele Fragen offen sind. Nach deren Klärung durch die SwissDRG AG wäre zu prüfen, ob diese Variante als alternative Übergangslösung angewendet werden könnte, bis die Güte der Tarifstruktur durch die weitere Ausdifferenzierung genügend ist. Eine Poollösung gemäss Lösungsansatz 4 dürfte hingegen schwer umsetzbar sein und ist eher ungeeignet.

\section{SwissDRG AG differenziert weiter aus - mit problematischer Hochdefizitfalldefinition}

Der Verwaltungsrat der SwissDRG AG hat sich für die weitere Ausdifferenzierung hochdefizitärer Fälle innerhalb der Tarifstruktur ausgesprochen, die FMH begrüsst diesen Entscheid. Weiter beschloss der Verwaltungsrat, dass auch Hochgewinnfälle berücksichtigt werden sollen und Varianten ausserhalb der Tarifstruktur aktuell nicht weiter verfolgt werden.

Dabei wendet die SwissDRG AG eine sehr restriktive Definition eines Hochkostenfalles für die SwissDRGVersion 6.0 an: Die Fälle werden als Hochkostenfälle anerkannt, deren Gesamtkosten mindestens 400000 Franken betragen und ein Defizit von mindestens 40000 Franken aufweisen - eine Definition, die wohl nur wenige Fälle erfüllen und überaus problematisch ist. Aus Sicht der FMH sind die Gesamtkosten für die Hochdefizitfalldefinition untauglich, relevant ist nur das Defizit.

Umso wichtiger ist es, bei der jährlichen Präsentation der neuen SwissDRG-Version zum Stand der Vergütungsproblematik hochdefizitärer Fälle durch die SwissDRG AG informiert zu werden. Dazu sind die bis dahin erzielten Wirkungen der getroffenen Massnahmen aufzuzeigen. In diesem Kontext wäre zudem wichtig, dass die SwissDRG AG auch Grenzen der Abbildungsmöglichkeiten von Hochdefizitfällen innerhalb der Tarifstruktur aufzeigt. Auch wenn der gewählte Weg noch keine abschliessende Lösung darstellt, gilt es diesen rasch und mit Entschiedenheit zu beschreiten.

Die detaillierte Stellungnahme der FMH zu SwissDRG und Hochdefizitfällen und den vorgeschlagenen Massnahmen finden Sie unter: www.fmh.ch $\rightarrow$ Stationäre Tarife $\rightarrow$ Positionen $\rightarrow$ Stellungnahmen. 